\section{Improved Risk Assessment of Tsunami Inundation Based on Geospatial and Local Knowledge for Sustainable Development}

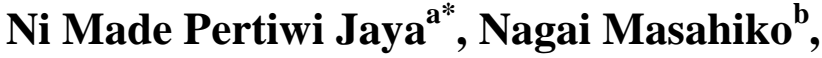 Gallardo Mauricio Reyes ${ }^{\mathrm{c}}$, Miura Fusanori ${ }^{\mathrm{d}}$}

\author{
a,b,d Space Utilization Engineering Laboratory, Graduate School of \\ Science and Engineering, Yamaguchi University, Japan \\ c Ocean Civil Engineering, Faculty of Engineering, University of \\ Valparaíso, Chile
}

Received: September3o, 2015 / Accepted: March 5, 2018

\begin{abstract}
Tsunami events have extensive implications for sustainable development, due to the social, economic, and environmental impacts they can cause. Take into account the competence of geospatial and local knowledge; these sources are integrated to assess tsunami risk assessment by utilizing satellite imageries. The study area of Talcahuano experienced severe damages due to the 2010 Chile earthquake and tsunami. The geospatial information of the damaged areas was identified by processing the surface reflectance values between pre- and post-disaster images of the satellite optical and SAR data. The DEM data was also employed to delineate areas according to the inundation height data. Local information of tsunami inundation was generated through a DIG participatory mapping. Both types of inundation maps showed good agreement with the results of field surveys. Furthermore, a map of the potential tsunami inundation zone was produced to aid integrated risk assessments in the area. The map showed areas with high, medium, and low inundation level adopted from the satellite images and the DIG map.
\end{abstract}

Keywords: Tsunami Inundati Satellite Imageries, Optical and SAR Sensor, DIG, Disaster Risk Reduction

\begin{tabular}{|lcl|} 
Abbreviations: \\
\hline DRR & $:$ & Disaster Risk Reduction \\
GIS & & Geographical Information System \\
ALOS & $:$ & Advanced Land Observing Satellite \\
VHR & $:$ & Very-High-Resolution \\
NIR & $:$ & Near Infrared \\
AVNIR-2 & $:$ & Advanced Visible and Near Infrared Radiometer \\
& & Type-2 \\
SAR & $:$ & Synthetic Aperture Radar \\
PALSAR & $:$ & Phased-Array L-band Synthetic Aperture Radar \\
DEM & $:$ & Digital Elevation Model \\
GDEM & & Global Digital Elevation Model \\
DIG & $:$ & Disaster Imagination Game \\
\end{tabular}

\section{Introduction}

The number of recorded natural disasters has risen globally in the course of the past decades, and their impact on human society has also increased, affecting several hundred million people annually [1]. A single disaster event can sometimes destroy a considerable proportion of the Gross Domestic Product (GDP) in many countries [2]. In 2011, the losses caused by natural disasters reached a record of US $\$ 371$ billion [1]. Tsunamis are one of the main types of disaster hazards [3]. Since the 2004 Indian Ocean Tsunami, the awareness of tsunami risks has been increasing worldwide [4]. Despite the relative low frequency (compared to other disaster events) tsunamis have the power to cause massive loss of life, significant economic losses, and cascading effects from the destruction of critical facilities [5].

The February $27^{\text {th }}$ Chile's Maule M8.8 Earthquake, which triggered a tsunami, produced the largest insured losses of 2010 [6]. This earthquake was the fifth largest earthquake measured in history [7]. As Chile is located on the so-called "Pacific Ring of Fire," it has been affected by thirteen earthquakes with magnitudes equal to or greater than 7.0 since 1973 [5]. The tsunami generated by the 2010 Maule earthquake was also the most intense in this period, with 2 to 11-meters waves hitting coastal towns for periods of up to four hours [8]. It caused severe damage to 45 coastal towns, with total economic damage of US $\$ 30$ billion [9]. The government reported casualties due to the earthquake and tsunami totaled 521, with a fourth of them being tsunami-related [8].

When attempting to reduce disaster losses, as stated on Sendai Framework for Disaster Risk Reduction (DRR) 2015-2030, the first of the four priorities of DRR is the understanding of disaster risk [10]. This is an essential requisite for the sustainable development of disaster-prone areas [11]. The improvement of risk assessments deals with the provision of an adequate disaster-related information that can contribute to the DRR [12]. Regarding this matter, there is a need to address existing challenges and prepare for future ones by focusing on risk assessment based on damage identification, as well as the dissemination of such information to the community.

According to the Integrated Coastal Area Management (ICAM) guidelines, the utilization of satellite remote sensing imageries through GIS technology is appropriate for estimating damage due to hazards, such as inundation areas [13]. Satellite data can be used for assessing risks promptly, as well as assisting rehabilitation plans [14]. Visual interpretation of inundated areas can be performed using Very-High-Resolution (VHR) optical satellite imageries [15], which can provide a high accuracy in the identification of affected areas [16]. Various types of Synthetic Aperture Radar (SAR) satellite imageries have also demonstrated a powerful ability to identify tsunami damage, i.e., L-band Advanced Land Observing Satellite (ALOS) Polarimetric SAR (PolSAR) [17], C-band ENVISAT Advanced SAR (ASAR) [18], and C-band TerraSAR-X imageries [19]. Particularly in Chile, tsunami-damaged areas have been detected by employing ALOS Phased-Array Lband SAR (PALSAR) imageries [20]. These identifications were carried out by obtaining the backscattering intensity of SAR data before and after the disaster.

Corresponding Author

E-mail: madepertiwi@yahoo.com 
Considering the possibility of damage detection by integrating two or more different types satellite data, research has been performed using optical, SAR, and DEM data. The optical data of the Advanced Spaceborne Thermal Emission Reflectance Radiometer (ASTER) and the DEM data was used to delineate the inundation line on SAR data of ENVISAT in tsunami-damaged areas [21]. Satellite missions, such as ALOS operated by Japan Aerospace Exploration Agency (JAXA), have developed the capability of satellite-derived imageries.

In addition to providing a damage assessment, satellite-based analysis results can be used as an input for creating disasterrelated risk maps, together with other historical data. Based on a global stakeholder assessment, end-users attributed a high operational benefit of inundation maps to design and operate critical infrastructure and provide humanitarian aid [12]. Therefore, an inundation map can be an important tool to support the dissemination of disaster-related information to the community. This sort of disaster knowledge represents one of the eight fundamental elements of coastal community resilience, as identified by the United States Agency for International Development (USAID) guide to reduce risk from coastal hazards, accelerate recoveries, and adapt to changes [22]. Despite the challenges associated with it, participatory mapping is valuable for improving community understanding of a certain disaster [23]. A Disaster Imagination Game (DIG) is an easy and cost-effective participatory mapping methodology invented in 1997 in Japan [24]. In the context of disaster risk reduction, it can be very empowering at the community level, since it enables an assessment based on people's perception of risks and coping strategies [25].

Regarding the the achievements of sustainable development that is associated with DRR, this study concerns itself with the improvement of risk assessments through geospatial and local knowledge. Taking into account the functionality and performance of satellite data on damage assessment, satellite optical and SAR imageries were utilized to identify inundation areas due to the 2010 Chile tsunami event. These damaged areas awerere extracted using the pre- and post-disaster images of the DigitalGlobe VHR optical data, ALOS AVNIR-2, ALOS PALSAR, and DEM data. The results were verified using land classification and field observation data, showing good agreement with the analysis of the satellite data. In order to generate local knowledge amongst coastal resident, a local community's understanding on tsunami damage was assessed through a map-based DIG participatory workshop. The results of the exercise indicate that the integration of the geospatial and local knowledge for assessing tsunami inundation zones can be a valuable tool to ensure sustainable development by reducing local risks and improving the resilience of the local population.

\section{Methodology}

\subsection{Case Studies}

The study was performed in Talcahuano, Chile (Figure 1). Geographically, this settlement lies at latitude $36^{\circ} 43^{\prime} 29.22$ "S and longitude $73^{\circ} 7^{\prime} 1.13^{\prime \prime} \mathrm{W}$. The area is located within the boundary of the Greater Concepcion area [8]. It is the port city in Biobio Region, which contains a naval base and has a population of around 250,000 inhabitants, based on the 2002 census [7]. It was also one of the main regions affected by the 2010 tsunami. The area of Concepcion is considered to be highly vulnerable to earthquakes and tsunamis [26]. A megathrust earthquake of M8.8 took place on the coast of the Maule Region of Chile on 27 February 2010 at 03:34 local time (o6:34 UTC) [7]. The earthquake generated a destructive tsunami, which is considered to be the biggest tsunami disaster in South America since 1979, and which propagated all the way to
Japan [27]. The area of Talcahuano in Figure 1 was generated using the natural color of the optical satellite imagery of ALOS AVNIR-2, showing also a map of the regions damaged by the 2010 Chile earthquake and a graph of tsunami inundation height and flows depth in the study area. This data are based on field survey reports $[7,26]$.

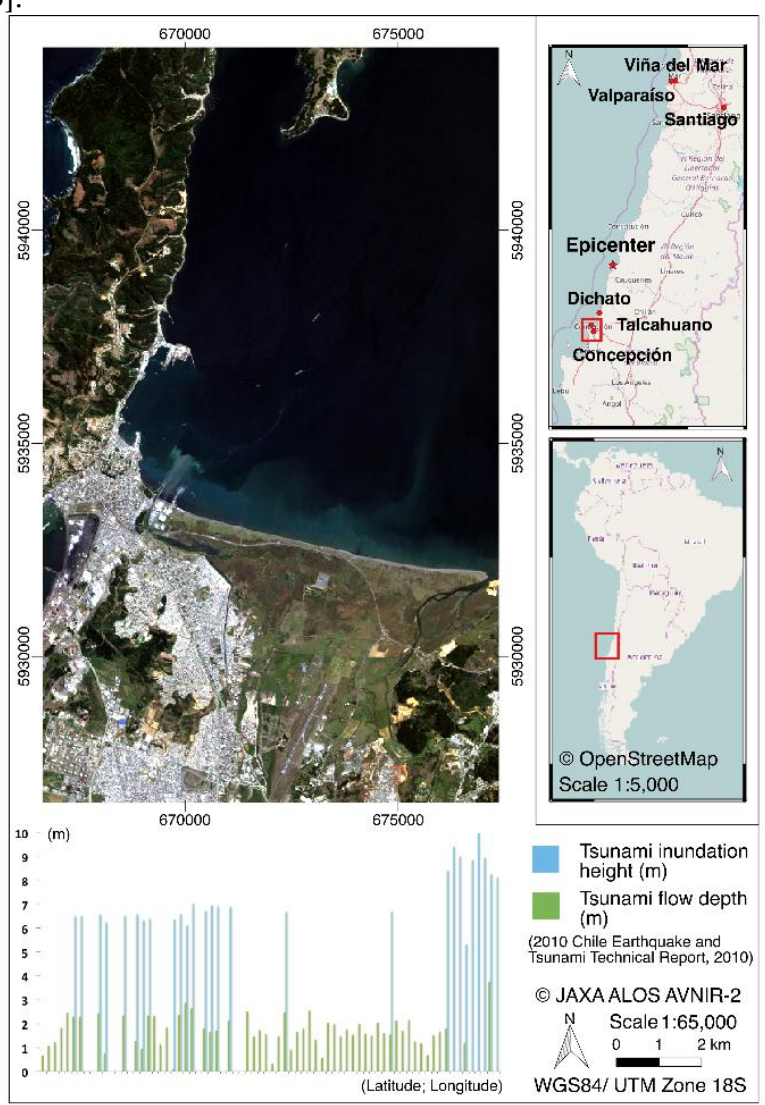

Figure 1 Study area of Talcahuano city

CATOE (Centro de Alertamiento Temprano y Oficina de Emergencia) reported that the tsunami attacked the coast at least four times within a period of 45 to 60 minutes, with the fourth wave being the largest [7]. The tsunami inundation heights measured in Talcahuano ranged from o to $10 \mathrm{~m}[7,26]$. The tsunami penetrated approximately $300 \mathrm{~m}$ inland in the port-town areas and more than $1 \mathrm{~km}$ in the southern coastal marshes. Tsunami inundation heights reached $6-8 \mathrm{~m}$ in the port-town area and $10 \mathrm{~m}$ in the coastal marshes. It damaged houses and infrastructures, including a bridge and sewerage pipelines in the east part of Talcahuano [28].

\subsection{Data Sources and Instruments}

The primary data for the study were satellite images. The satellite data (Table 1) was comprised of optical data, SAR data, and DEM data. The satellite data processing results were validated using secondary data, which consisted of tsunami inundation maps [40, 41], field survey reports on inundation height $[7,26]$, and damage observation results due to the 2010 Chile earthquake and tsunami event [28]. Moreover, community-based information on DIG maps related to the tsunami inundation in Talcahuano were used as inputs for the analysis. The data processing for integrating the information was performed using image processing and geospatial analysis software, i.e., Environment for Visualizing Images (ENVI) and Quantum GIS (QGIS). 
Table 1 List of satellite data used in the study

\begin{tabular}{|c|c|c|c|}
\hline $\begin{array}{l}\text { Satellite } \\
\text { product }\end{array}$ & $\begin{array}{l}\text { Sensor or } \\
\text { Data Type }\end{array}$ & $\begin{array}{c}\text { Acquisition } \\
\text { Date }\end{array}$ & Resolution \\
\hline GeoEye-1 & VHR Optical & $2009 / 02 / 08$ & $2.44 \mathrm{~m}$ \\
\hline Quick Bird & VHR Optical & $2010 / 03 / 19$ & $1.65 \mathrm{~m}$ \\
\hline ALOS & High-Resolution & $2007 / 02 / 24$ and & \\
\hline AVNIR-2 & Optical & 2010/04/o9 & $10 \mathrm{~m}$ \\
\hline ALOS PALSAR & SAR & $\begin{array}{r}2009 / 03 / 11 \text { and } \\
2010 / 03 / 14\end{array}$ & $6.25 \mathrm{~m}$ \\
\hline ASTER-GDEM & DEM & 2007 & $30 \mathrm{~m}$ \\
\hline
\end{tabular}

\subsection{Research Framework}

An assessment of hazard risks is necessary to accomplish the sustainable development of an area [29]. A short-term assessment of a major natural hazard is a difficult task, considering that the impacts may vary according to the spatial scale. Nevertheless, recent studies have highlighted a variety of ways in which geospatial information systems can contribute to risk reduction practices [30]. Based on a global stakeholder assessment, in the case of tsunami hazards it appears that the inundation zone is one of the most important geospatial information criteria for assessing the risk. A tsunami inundation hazard map can be created by combining the results of satellite data analysis with other tsunami inundation related information from the local community. Aside for constituting a tool for investigating community knowledge on disasters, a participatory mapping exercise can extensively contribute to the collection of information. Therefore, the framework of the research (Figure 2) aims to achieve a holistic understanding of the risks to a given community through satellite data processing and community-based participatory mapping, in order to improve the resilience of the community to tsunami hazards.

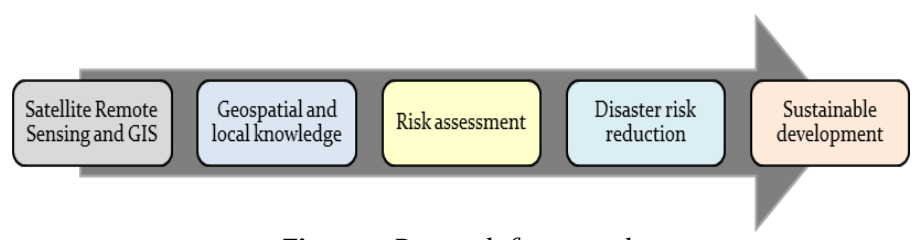

Figure 2 Research framework

\subsection{Research Scheme}

According to the framework of the study, the methodology used in the research (as described in the flowchart below, see Figure 3) consists of two main processes, i.e., satellite data analysis and map-based DIG participatory mapping. The evaluation, using observation data, is the subprocess for both of the main processes. A map of tsunami inundation zone was finally produced through an integrated assessment of the tsunami hazard risk through the analysis of satellite imageries and the information obtained from the community.

\subsection{Data Analysis}

The data analysis was comprised of the analysis of the satellite data and a DIG participatory mapping exercise.

\subsubsection{Image Processing and Geospatial Analysis}

Fundamentally, there are three points in this process, i.e., image rectification and restoration, image enhancement, and information extraction. However, variation and modification can be applied depending on the data quality and methods available.

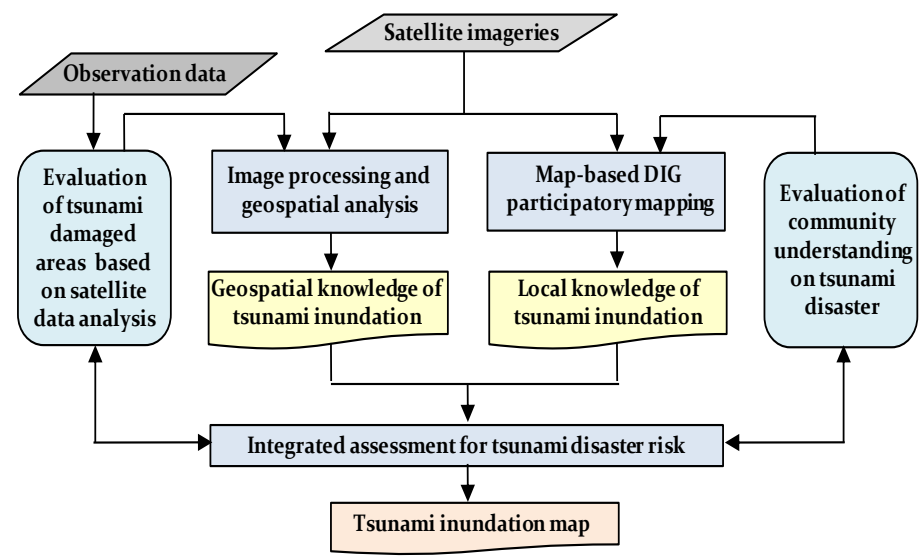

Figure 3 Research methodology for the improvement of tsunami risk assessment

Prior to the extensive image processing of tsunami-damaged areas, a qualitative observation through visual interpretation of damaged locations was carried out. Based on research conducted using VHR satellite optical data in three different areas, visual interpretation of the pre- and post-disaster images has produced excellent results for tsunami damage assessment [15]. In this study, the VHR satellite optical imageries were visualized through the natural colour composite of a panchromatic sharpened image.

A panchromatic sharpened image represents a sensor fusion between the high spectral resolution of the multispectral and panchromatic images [30]. The Gram-Schmidt method, based on a general algorithm vector orthogonalization, was used for the panchromatic sharpening, which is necessary for the VHR optical data processing to generate a high spatial and spectral resolution image. Band composite represented the natural colour, which was applied afterwards to produce colours which would resemble those that would be observed by the human eye. The colour channels were displayed in red, green, and blue (RGB) by combining three different wavelength regions (band) of the data [31]. The natural colour composite of the GeoEye-1 (pre-disaster) and Quick Bird (post-disaster) were created using the combination of band 3, 2, and 1, which has the spectral range of red, green, and blue, respectively (Figure 4). The results were compared to identify the initial tsunami-damaged areas.

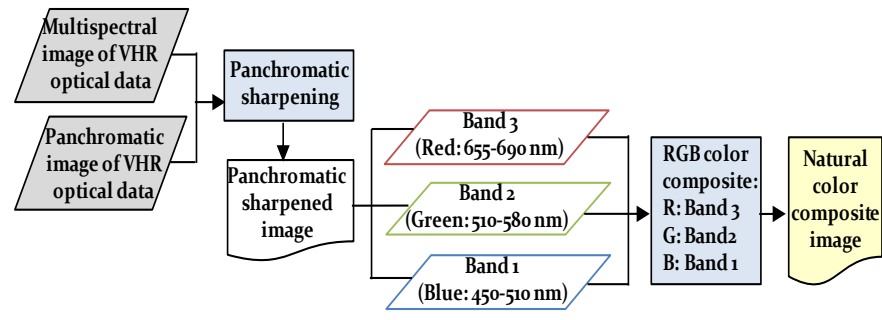

Figure 4 Image processing of the VHR satellite optical imageries

Furthermore, a quantitative analysis can be performed by calculating the measured values of the satellite data. In the case of the optical imagery, the data values are recorded by a passive sensor, which functions similarly to a human eye, by recording non-polarized sunlight reflectance of the surface materials with a large range of different wavelengths [31]. A raw image was produced by converting at-sensor radiance measured in the wavelength region to Digital Numbers (DN) using a quantification system. This contains the effects of the light source, sensor, atmosphere, and surface material without any unit or physical connotation. Therefore, the raw image needs to be corrected by calibrating the $\mathrm{DN}$ into radiance. The following equation describes the algorithm for the conversion of DN to radiance [32]. 
$L_{\lambda}=G_{\text {rescale }} \times Q C A L+B_{\text {rescale }}$

Where, $L_{\lambda}$ is the spectral radiance at the sensor's aperture $\left(\mathrm{W} / \mathrm{m}^{2} / \mathrm{sr} / \mu \mathrm{m}\right), G_{\text {rescale }}$ is the rescaled gain, $Q C A L$ is the $\mathrm{DN}$, and $B_{\text {rescale }}$ is the rescaled bias. The rescaled gains and biases for ALOS/AVNIR-2 are shown in Table 2. The radiance was then corrected to obtain the top-of-atmosphere (TOA) reflectance. It provides acceptable information of surface materials, e.g. vegetation, water, and other areas. Equation 2 describes the algorithm for the conversion of radiance to reflectance [33].

$\rho_{\lambda}=\pi \times L_{\lambda} \times d^{2} / \operatorname{ESUN}_{\lambda} \times \cos \theta_{s}$

Where, $\rho_{\lambda}$ is the unitless planetary reflectance, $L_{\lambda}$ is the spectral radiance at the sensor's aperture, $d^{2}$ is the Earth-sun distance in astronomical units from a nautical handbook, $E S U N_{\lambda}$ is the mean solar exoatmospheric irradiances, and $\theta_{s}$ is the solar zenith angle in degrees.

Table 2 Calibration factors of spectral reflectance for the ALOS AVNIR-2

\begin{tabular}{lrrrrr}
\hline \multirow{2}{*}{ Bands } & \multicolumn{2}{c}{ Wavelength } & $\boldsymbol{G}_{\text {rescale }}{ }^{\left[{ }_{32}\right]}$ & $\boldsymbol{B}_{\text {rescale }}{ }^{\left[{ }_{32}\right]}$ & ESUN $^{[33]}$ \\
\hline regions $(\mu \mathrm{m})^{\left[{ }_{33}\right]}$ & $0.42-0.50$ (blue) & 0.5888 & 0 & 1943.3 \\
Band 2 & $0.52-0.60$ (green) & 0.5730 & 0 & 1813.7 \\
Band 3 & $0.61-0.69$ (red) & 0.5020 & 0 & 1562.3 \\
Band 4 & $0.76-0.89$ (NIR) & 0.8350 & 0 & 1076.5 \\
\hline
\end{tabular}

An analytical process to identify changes on land or water surfaces was conducted after quantitatively interpreting an image from the satellite data. In this regard, the difference in the vegetation index, or Normalized Difference Vegetation Index (NDVI), is one of the common methods used. A study after the 2004 Indian Ocean tsunami, using various moderate- to highresolution optical satellite imageries before and after the event, demonstrated that the tsunami-affected areas could be identified from the NDVI [16]. Therefore, in the present work high-resolution satellite optical data of ALOS AVNIR-2 before and after the disaster was employed to identify the damaged areas. The NDVI pre- and post-disaster were calculated after performing calibration and atmospheric correction of the raw images. NDVI, as explained in Equation 3, essentially measures the reflectance difference between two certain wavelength ranges from -1 to 1 [34].

$\mathrm{NDVI}=\frac{(N I R-V I S)}{(N I R+V I S)}$

Where NIR is the reflectance of near infrared band and VIS is that the visible band of red band of ALOS AVNIR-2. In particular, band 4 is NIR, and band 3 is red (Table 2 ). Besides observing changes in pre- and post-disaster images, both of the NDVI values were subtracted to determine a boundary value that could be used to divide the post-disaster image into inundated areas and those that were not. Areas damaged as the result of tsunami inundation lie in the water areas of the image. The inundated area can be calculated by subtracting the pre-disaster water areas from the post-disaster water areas. Figure 5 describes the flowchart of the image processing of ALOS AVNIR-2 imageries.

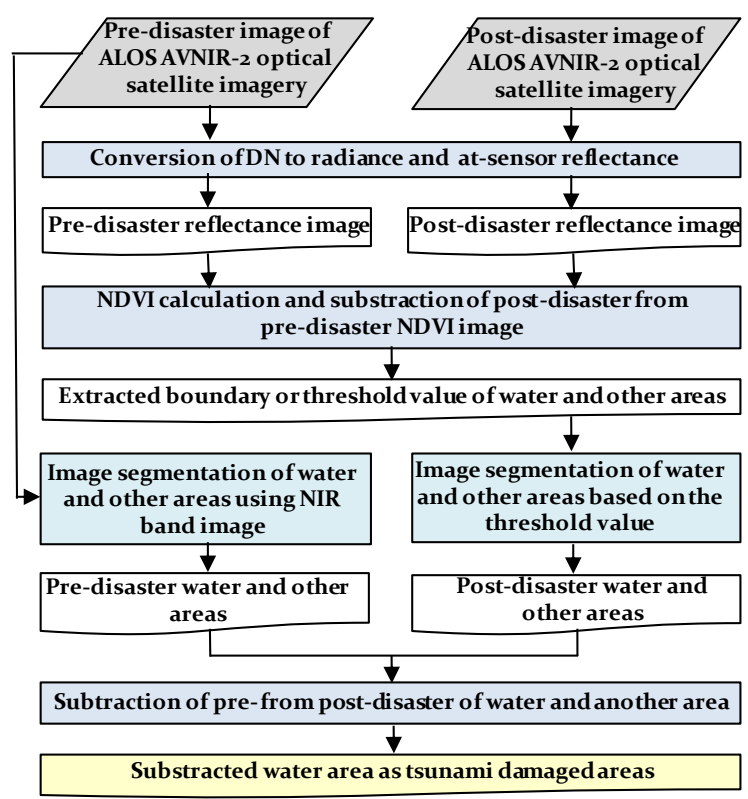

Figure 5 Image processing of the extraction of tsunami-damaged areas using ALOS AVNIR-2 imageries

Additionally, the SAR data of ALOS PALSAR were processed to extract tsunami-damaged areas. Figure 6 describes the flowchart of the image processing. One of the advantages of using radar is that the sensor is not affected by the weather and day and night cycles [35]. Unlike the optical imagery, the SAR imagery is generated by an active or radar sensor. It emits a light pulse and records the part of the pulse, which is reflected, or scattered, back to the sensor, referred to as backscatter. The radar is a laser, which operates within a narrow and well-defined wavelength bands at a specific polarization. It technically works as a flash camera in a dark room [36]. The wavelength band strongly affects the sensitivity of the radar to the size of objects. It recognizes objects, which have the same and larger spatial magnitude than the radar wavelength. On the other hand, objects smaller than the radar wavelength has less influence or become transparent on the backscatter [37]. For instance, ALOS PALSAR operates within the L-band, which has a long wavelength radar signal of $23.5 \mathrm{~cm}$. Therefore, it penetrates through the forest canopy and interacts with the larger structures, such as the trunks and the larger branches of trees. Systems operated at shorter wavelengths are more sensitive to sparse and low vegetation [38]. Moreover, the strength of the backscatter is affected by the radar polarization. Currently, the radar signal of spaceborne radar systems operates with linear polarization. It is transmitted and received at horizontal $(\mathrm{H})$ and vertical (V) polarization [31].

The radar backscatter per unit area or $\sigma^{\circ}$ (sigma-naught) represents the radar image brightness, which has a unit of $\left[\mathrm{m}^{2} / \mathrm{m}^{2}\right]$ expressed in decibel $(\mathrm{dB})$. The $\sigma^{\circ}$ varies even for homogeneous targets depending on the incidence angle, and the angle between the ground and the sensor. In this case, normalization of the $\sigma^{\circ}$ on the incidence angle can remove the range dependency to eventually obtain a normalized $\sigma^{\circ}$ or $\gamma^{\circ}$ (gamma-naught) [36]. The DN on raw images acquired particularly from ALOS PALSAR data can be converted to $\gamma^{\circ}$, as described in Equation 4, using a calibration factor $(\mathrm{K})$ of $-83.0 \mathrm{~dB}$, as provided by JAXA [38].

$\gamma^{\circ}=10^{*} \log _{10}\left(D N^{2}\right)-83[d B]$

(4) 


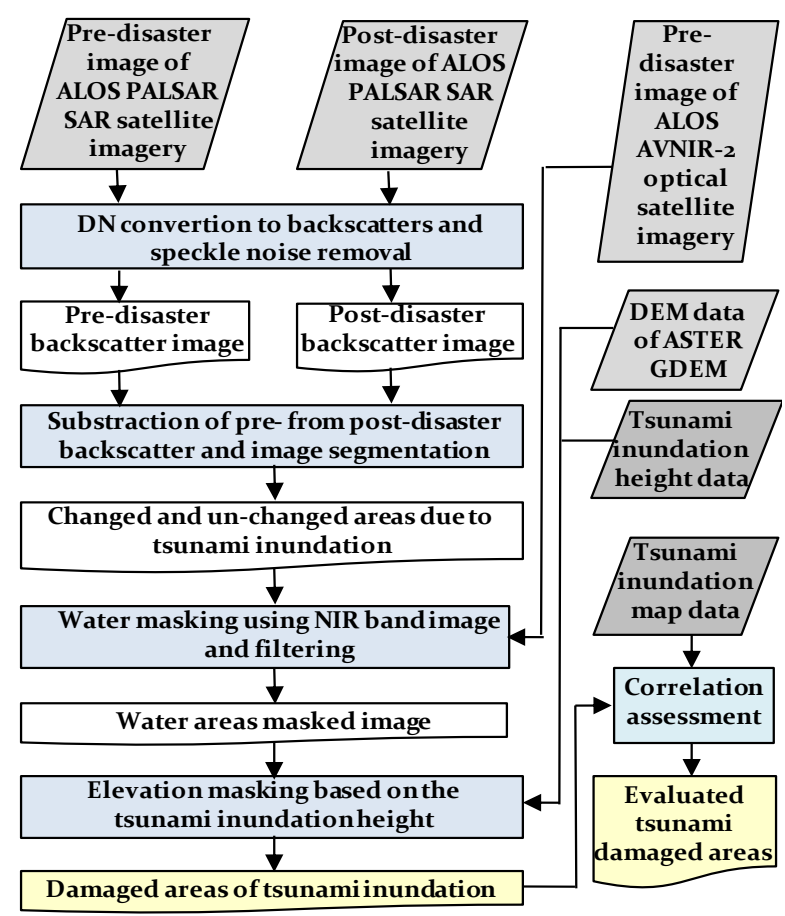

Figure 6 Image processing of the extraction of tsunami-damaged areas using ALOS PALSAR imageries

Regarding tsunami disaster monitoring, the backscatter difference between pre- and post-disaster images generated from SAR data in different wavelengths have been successfully utilized to detect damage or inundation areas $[17,18,19]$. Taking into account the ability of backscatter for this purpose, in the present work it was applied to the pre-processing. A process of removing speckle noise from the backscattered image results was carried out before the backscatter calibration. The speckle noises were removed using a Lee filtering method to reduce the interferences on the image acquired by the radar image, as well as soften the image. A segmentation threshold value of changed and unchanged area values were obtained based on the subtraction result of the post-disaster from the pre-disaster backscatter. The changed area values were obtained initially as tsunami damage areas.

Afterward, the damage analysis was conducted more thoroughly on the analysis processing. Masking is the main process of this step, which was undertaken through the use of other satellite data. Fundamentally, a mask is a raster image that consists of the values of 1 and $o$, which denote the active pixels and the masked pixels, respectively. By multiplying the mask onto an actual image, masked pixels are screened out from the image, so that the image only has the desired pixels [31]. In this case, a water mask from the NIR band images of ALOS AVNIR-2 and an elevation mask of the tsunami inundation height from the DEM data of ASTER GDEM were created for the analysis processing.

Water areas can be observed adequately through the NIR wavelength of the optical imageries, such as ALOS AVNIR-2. The water mask is used to eliminate the water areas, e.g., sea water, river, lake, pond, and so forth, obtained by the optical sensor on the post-disaster image of ALOS PALSAR. Therefore, the resulting image only contains values of inundation water, and not actual water areas, and thus represents the extent of the tsunamidamaged areas. A median filtering method was then applied to the result to reduce disturbances in the image.

In the case were information regarding the tsunami inundation height is available, this information can be used to build an elevation mask covering the areas with the range of the inundation height. Based on the field survey data, the tsunami inundation due the 2010 event reached $0-10 \mathrm{~m}$ in the area of Talcahuano $[7,26]$. The elevation mask was applied to the filtered water masked image to create a so-called active area which denotes tsunami-damaged areas. These areas are water areas with an elevation range similar to the tsunami inundation height.

The post-processing was initiated by the morphological transformation and followed with the area calculation. The morphological processing was conducted to diminish or expand certain pixels on the image. Lastly, the two main segmented areas of the image, i.e., damaged and undamaged areas were calculated, to measure the total dimensions of the inundated and noninundated. These areas were evaluated according to the land classification generated from the optical data of ALOS AVNIR-2 and the inundation map produced by the related institution in Talcahuano, Chile.

\subsubsection{Map-based DIG Participatory Mapping}

DIG was invented in 1997 in Mie Prefecture, Japan. It is an easy and cost-effective methodology of Table Top Exercises for disaster management [24]. There are two stages that should be considered when performing DIG (Figure 7), i.e., the pre-event or preparation stage and the event stage. The DIG was performed as part of the training on Communitarian Management of Disaster Risks in Talcahuano, and was organized as a cooperation exercise between Chilean and Japanese institutions, i.e., Ingenieria Civil Oceanica of the Universidad de Valparaiso Chile, JICA, and Yamaguchi University.

A lecture was provided as an introduction to the theme of the study. Then, a handout with instructions for participants was provided for the mapping exercise, as well as the evaluation. Items, process steps, legends, and the instruction used for the hand out (Table 3) were translated into Spanish. Participants were grouped into teams, and each team was requested to draw the information mentioned on the hand out onto a base map of the area and present the results afterward. The initial and final evaluation of the exercise was assessed by participants through a self-evaluation activity.

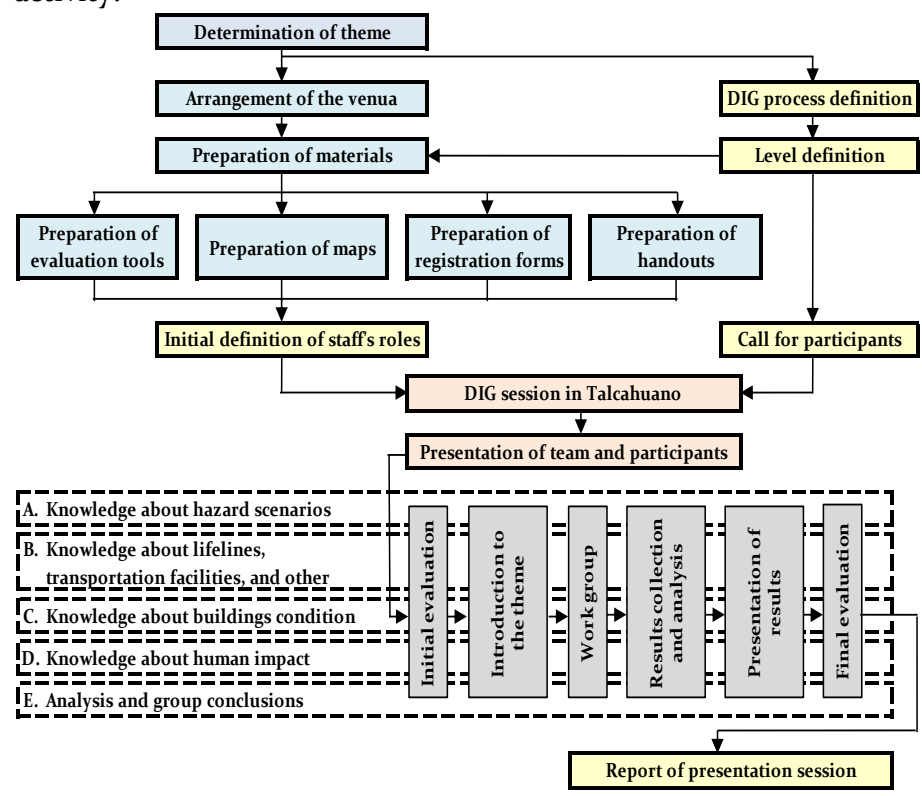

Figure 7 DIG flowchart, which comprises pre-event and event stages

There are five items which were evaluated by the DIG, i.e., knowledge about hazard scenarios, knowledge about lifelines, transportation facilities, and other, knowledge about the condition of buildings, knowledge about human impacts, and analysis and group discussion. According to the process of the hazard scenario item on the DIG hand out (Table 3), this step evaluates the community's understanding of the shoreline, inundation line, and inundation sequence. Further, such information was also utilized as the input for mapping the tsunami inundation zone. 
Table 3 The item, process step, and evaluation of DIG

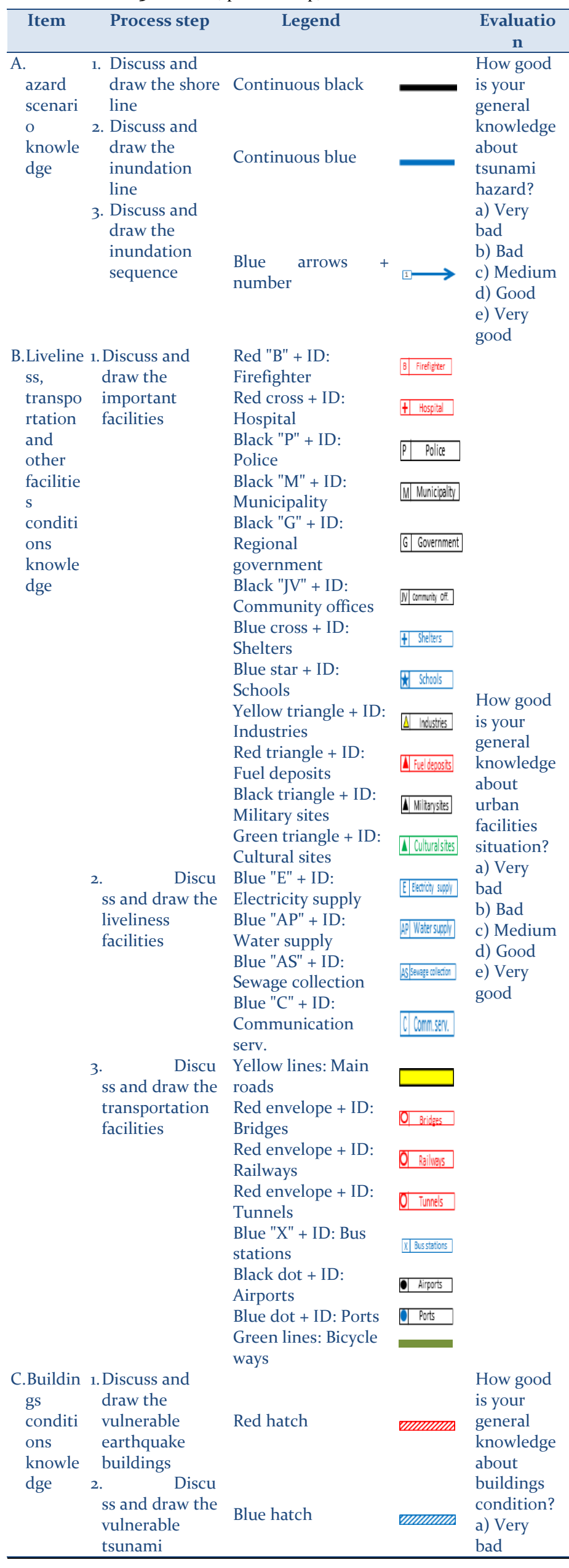

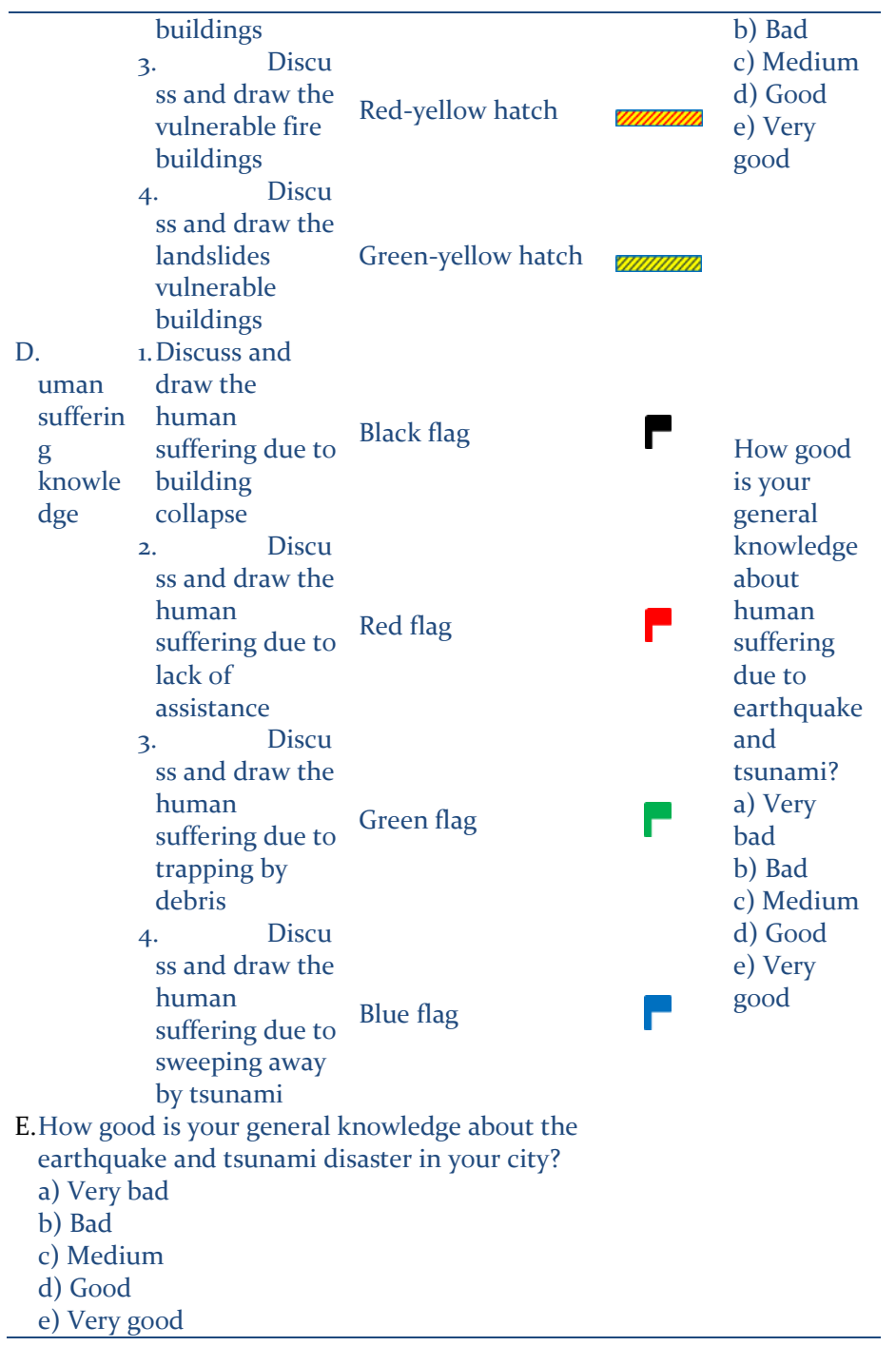

\section{Results}

The analysis of the data provided the extend of the damaged areas (from the satellite data) and other information related to the tsunami inundation.

\subsection{Geospatial Knowledge of Tsunami Inundation}

The locations that were damaged due to the 2010 tsunami inundation in Talcahuano area could be observed from the visual interpretation of the pre- and post-disaster images of VHR optical data. Objects could be recognized in detail from the panchromatic sharpened images, with resolutions ranging from 1.65 to $2.44 \mathrm{~m}$. The natural color composite of the images appropriately represented the colors of the surface materials. The extension of the damage was obtained by comparing the condition of several points before and after the disaster. Based on this identification, the damaged (Figure 8) areas encompassed the shoreline, the port area, and housing areas, which correlates well with field survey reports [28]. 


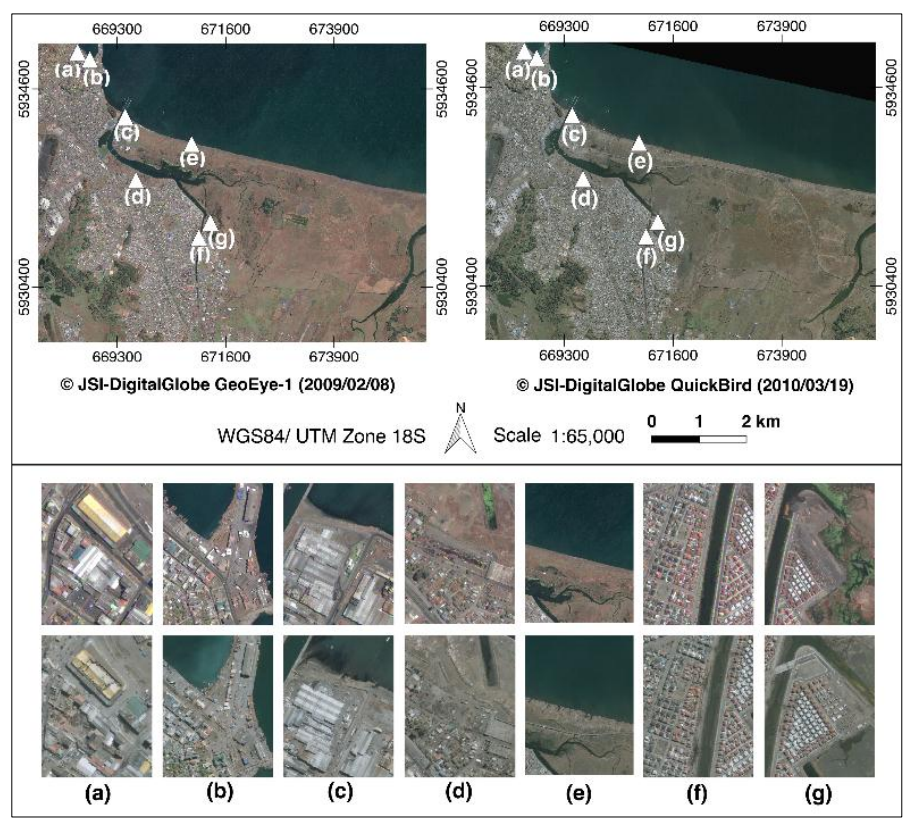

Figure 8 Visual interpretation of damage locations due to tsunami inundation from pre- and post-disaster images of VHR satellite optical data

Furthermore, the tsunami-damaged areas (Figure 9) were quantitatively assessed based on the NDVI difference before and after the event. The NDVI ratio makes use of the NIR and visible band of red acquired by the optical sensor. Technically, the sensor forms images of targets on the ground, which reflect and absorb the solar radiation detected by the sensor at a different wavelength Thus, surface materials such as water or inundated areas can be differentiated by their spectral reflectance signatures. It is also important to calculate the NDVI based on the converted reflectance values from the $\mathrm{DN}$ of the raw image. The values express the reflection from objects recorded by the optical sensor of the satellite. Based on thid processing, the NDVI difference in values between the post and pre-disaster images range from 1 to -1. Fundamentally, the NDVI values of water areas are expressed in negative values below o, while dense vegetation has values of more than 0.5 [34]. By subtracting the pre- and post-disaster image, the areas which still had NDVI water values on the post-disaster image represented the tsunami inundation areas. Such areas were observed around the coastal area, housing area, built-up areas such as housing and airport, and water basins alongside rivers, representing a total area of 3,546.32 ha.

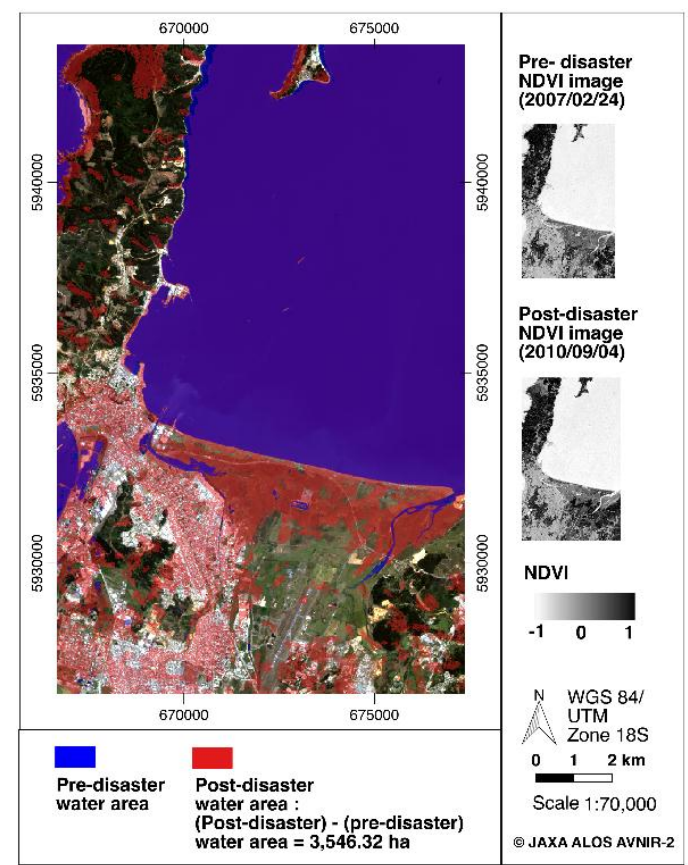

Figure 9 Water area changes based on NDVI difference of pre- and postdisaster images of ALOS AVNIR-2 optical data

Unlike optical data image, a more comprehensive image processing for obtaining damaged areas using images regards the use of ALOS PALSAR data. The backscatter was calibrated from the DN of the SAR data to obtain the actual values of reflected light pulses recorded by the SAR sensor. The frequency distribution of the backscatter absolute values from pre- and postdisaster was used to determine a segmentation threshold value. An image without water areas was produced afterwards by applying a water mask on the segmented image. Areas inundated by the tsunami were extracted after masking out areas with elevation beyond the tsunami inundation height of o-10 $\mathrm{m}$ (Figure 10). The inundated areas encompassed a total of 3,554.54 ha.

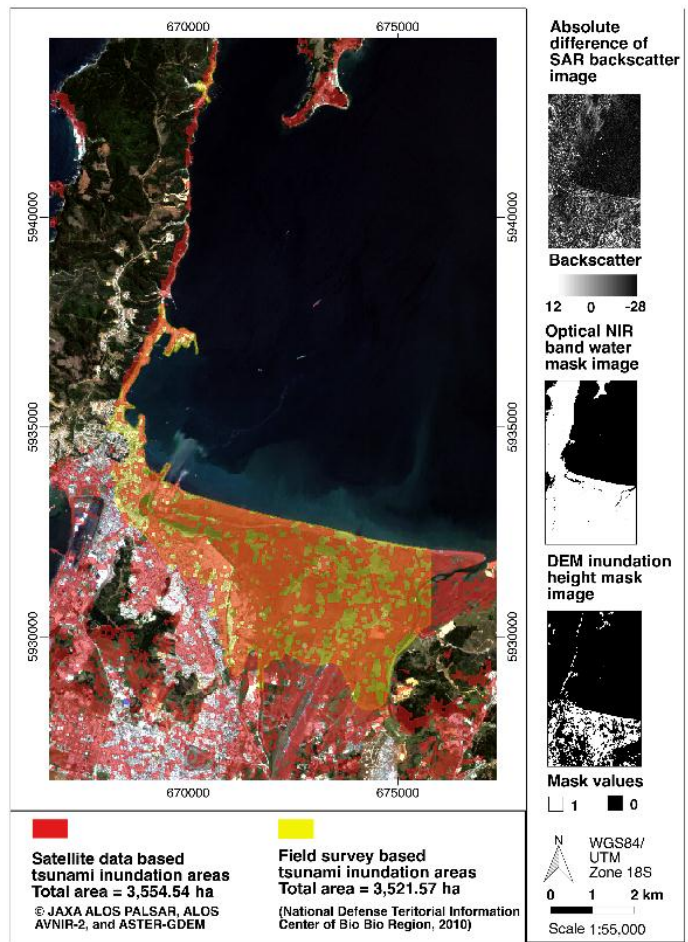

Figure 10 Inundated area according to the satellite data extraction and field survey reports 
Despite the fact that damaged areas were distributed in a disorderedly manner and covered an extensive terrain, the changes in surface reflection values generated from the pre- and postdisaster satellite data appeared to be good. The map produced by the local government indicated a similar distribution of tsunami inundated areas, which representing a total area of 3,521.57 ha [39]. Thus, a good agreement was attained, with the percentage of error difference between the satellite data extraction results and the observation results being around $0.70 \%$ for the ALOS AVNIR-2 and $0.93 \%$ for the ALOS PALSAR-2. Hence, it was possible to conclude that satellite imageries are adequate for estimating inundation areas after a tsunami.

Furthermore, the areas damaged by the tsunami inundation and the other areas, which encompass $68 \%$ of the total area of Talcahuano city, were evaluated according to land classification maps (Figure 11). The Chilean land classification system was used, which consists of five different general classes, i.e., wetland, water area, vegetation area, built-up area, and bare land [40]. A maximum likelihood method for supervised classification was applied to the ALOS AVNIR-2 optical data. The process was assisted with the panchromatic sharpened image of the predisaster GeoEye-1 VHR optical data as a reference.

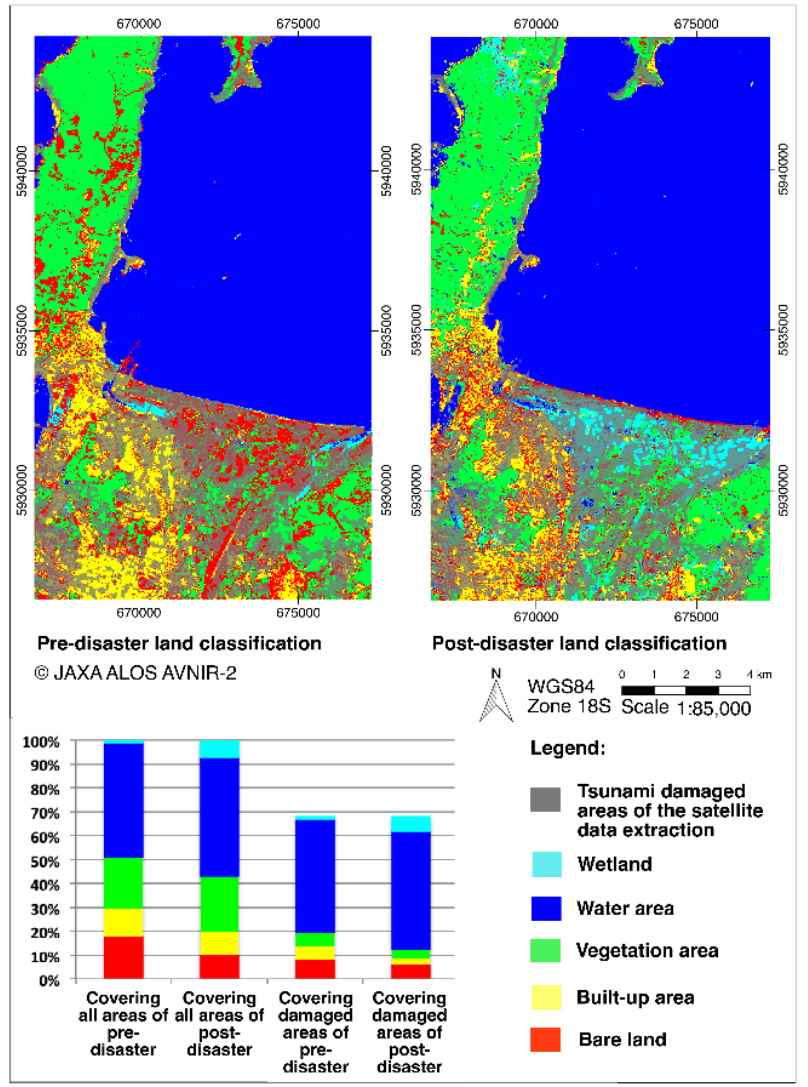

Figure 11 Comparison of the pre- and post-disaster land classification in the area of Talcahuano, Chile

The land classification result demonstrated that the areas classified as water area and wetland expanded in the post-disaster images, compare with the pre-disaster , by about $2 \%$ and $6 \%$, respectively. Otherwise, the bare land, built-up, and vegetation area diminished by more than $5 \%$. In agreement with the results, the satellite optical sensor detected that the earthquake and tsunami resulted in changes to the bare land, built-up area, and vegetation areas, which turned into wetland and water areas.

\subsection{Local Knowledge of Tsunami Inundation}

The DIG session was held in Talcahuano city on April $20^{\text {th }}, 2015$. There were 70 participants, consisting of professional and nonprofessional, who had been living and working in the area for at least 20 years. Participants were requested to divide themselves into seven groups. Each team wrote down information on a 1:25,00o scale map of the Talcahuano administrative area, which was covered with a transparent sheet. The information comprised the present location of strategic roads and bridges, rivers, and open places. Other important facilities and buildings were also confirmed on the map, such as a hospital, communication center, airport or churches. In particular, information regarding the tsunami inundation was also gained, based on the knowledge of the participants of the disaster event. Figure 12 shows how these participatory mapping activities were conducted.
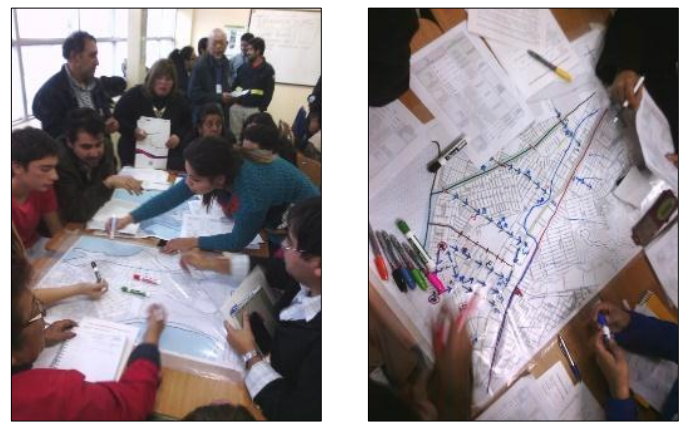

Figure 12 The DIG mapping activities in Talcahuano city

After participating in the DIG, an evaluation of the exercise was made by requesting participants to describe their knowledge of the disaster and other related aspects before and after the DIG activity, on a scale of o-5 (Table 3 ).

The DIG evaluation is shown in Figure 13, which indicatesa high knowledge about the hazard scenario, human impacts, and the earthquake and tsunami hazard. On the contrary, the knowledge about lifelines, transportation, and other facilities, as well as the knowledge about building conditions, were not as good. Moreover, the assessment was able to detect issues for future improvements in the DIG method. Knowledge about the hazard scenarios increased, and it thus seems that the DIG map is useful for improving knowledge, particularly of the shoreline, tsunami inundation, and tsunami sequence of events.

The map drawn by the participants of the DIG was modified and transformed into a tsunami inundation map, as shown in Figure 14. The information obtained from the community about the area was presented on the map, i.e., vegetation and built-up areas. The main information is related to the tsunami inundation, i.e., the primer or index curve and secondary curve of the inundation line. There are three levels of danger highlighted in this map, i.e., a high level for ground elevations between o to $0.5 \mathrm{~m}$, a medium level between 1 to $2 \mathrm{~m}$, and the low level for higher grounds above $2 \mathrm{~m}$. Moreover, the inundation level indicates the flow speeds. A high level indicates high flow speed, whereas a medium and low inundation level denote lower flow speeds. 


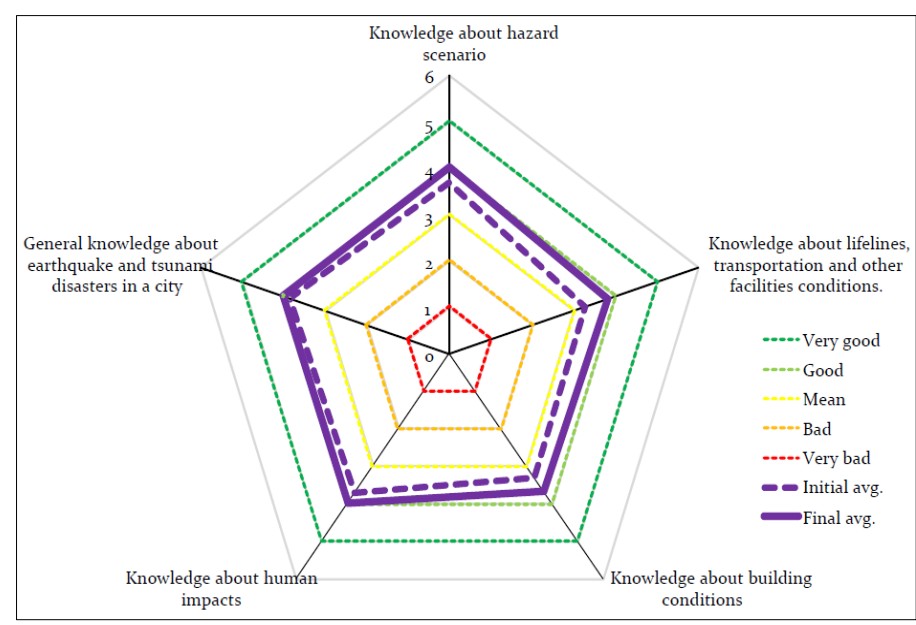

Figure 13 The evaluation result of DIG in Talcahuano city

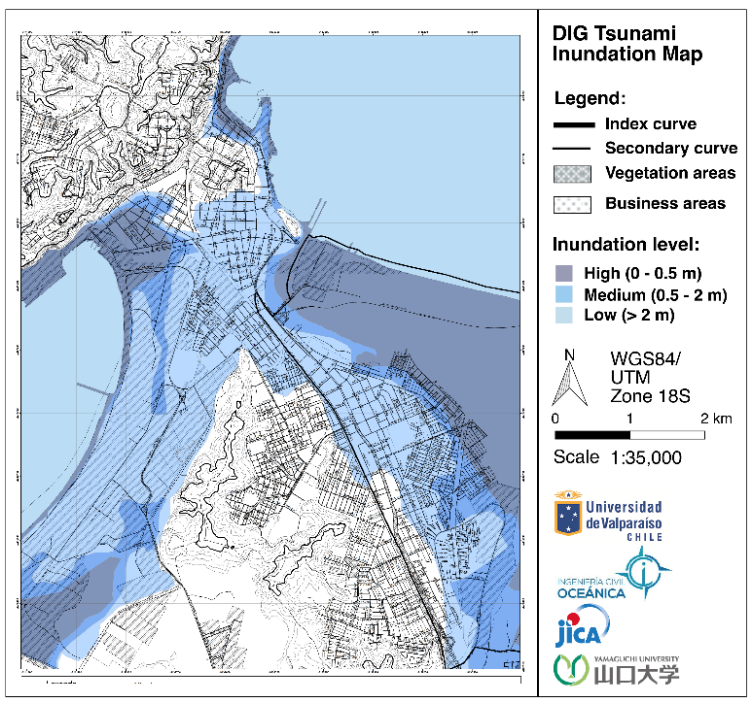

Figure 14 A modified map of tsunami inundation zone based on the DIG

\subsection{Integrated Risk Assessment of Tsunami Inundation Zone}

Finally, the quantitative data from the satellite data analysis was complemented with the qualitative data from the community DIG activity. The integration of such information can be considered an important tool to improve risk assessment of tsunami inundation. A tsunami inundation zone map was produced as the integrated risk assessment result (Figure 15). The map provides information regarding the level of tsunami inundation, which identifies the vulnerability of Talcahuano to tsunami events.

The map comprises three levels of inundation, following the DIG map. This classification further compiled and interpreted using the coastal proximity algorithm (Equation 5) into the distances from the shoreline, i.e., high (o to $0.5 \mathrm{~km}$ ), medium ( 0.5 to $2 \mathrm{~km}$ ), and high (above $2 \mathrm{~km}$ ).

$\log X_{\text {max }}=\log 1400+3 / 4 \log \left(Y_{0} / 10\right)$

Where Xmax is the maximum reach of the tsunami over land, and $Y_{0}$ is the height of the tsunami at the coast [42].

Regarding the classification of the land (Figure 11), segetation area and bare land along the coast are mostly classified as having a high level of inundation. Built-up area nearby the coast in El Morro District also have a high potential level of tsunami inundation. In the other districts, most of the built-up areas are on medium inundation level. The airport area is also within the tsunami inundation zone, with a low inundation level.

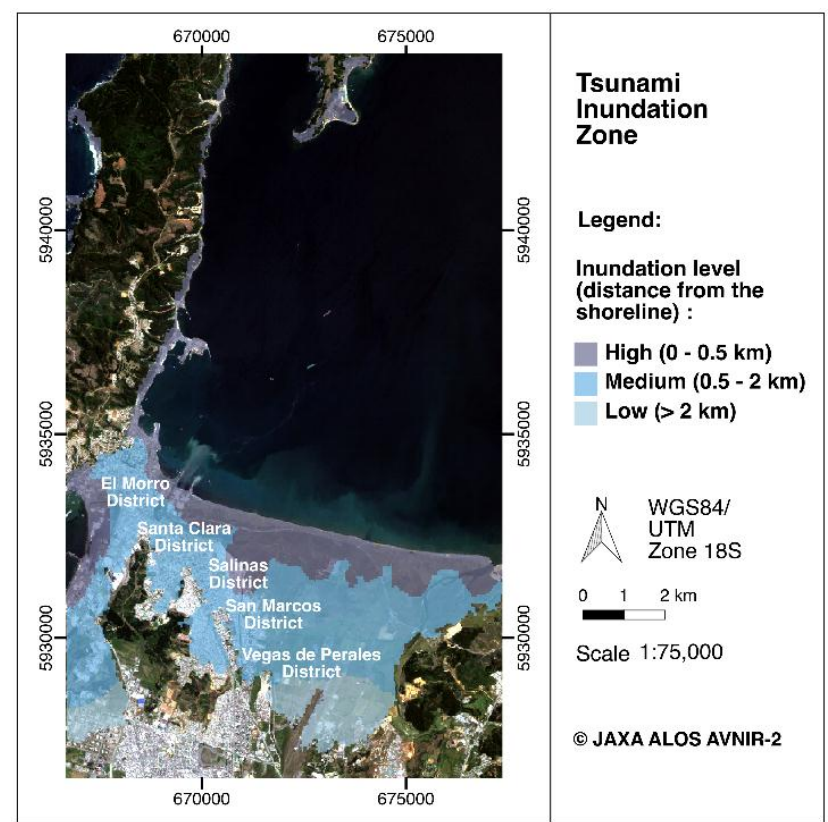

Figure 15 Tsunami inundation zone map based on the integrated geospatial and local knowledge assessment

\section{Discussion}

Tsunamis are considered to be one of the main types of hazards that can affect coastal areas [3]. Regarding sustainable development, disaster prevention measures are essential to reduce the disaster risk along coastal areas. Experiences over recent years regarding the impacts of tsunamis have shown that inadequate preparation for, and response to, emergency situations have contributed to widespread damage and the avoidable loss of lives and livelihoods [8]. In some instances, these shortcomings have been due to a poor regional identification of the risk as well as its dissemination to the community [6]. In this regard, it is crucial to identify and quantify tsunami disaster risks, particularly the potential extent of tsunami inundation [13].

Satellite remote sensing and GIS analysis are an advanced tool that can be used to carry out an identification of damaged areas after an event, which can serve as the foundation of risk assessment methodologies. A major reason for the adoption of remote sensing by risk management methodologies is that it is one of the fastest means of acquiring data for pre- and post-disaster studies, which can also assist rehabilitation plans [14]. The extraction result of damaged areas due to the 2010 tsunami and earthquake disaster in Chile using satellite data demonstrated a good agreement, when compared with the results of field survey observations. The processed images of optical data were qualitatively and quantitatively sufficient to be utilized as an initial rapid damage assessment. Moreover, the processing result using SAR, optical, and DEM data generated an adequate identification of tsunami inundation. Both the vegetation index of NDVI calculated from the image of ALOS AVNIR and the backscatter of the ALOS PALSAR were applicable to detect the damage due to the tsunami's inundation.

Due to high condensation during certain periods, optical data sometimes results in images which consist mostly of clouds, and in this case the use of SAR data con prove to be effective. In the last two decades, SAR has become the premier sensor to observe some phenomena due to its sensitivity to small surface roughness changes, and given that it is also independent of solar illumination and is unaffected by cloud cover [35]. In this regard, the SAR surfaces reflected light pulses and were able to extract changed and unchanged areas. Subsequently, the inundated areas were 
determined using the optical and DEM data to delineate the water and elevation of inundation, respectively.

However, when attempting to compute the extent of the damage it is important to consider the difference in time between the event and the time when the satellite images are taken [14]. The identification of areas damaged by the 2010 Chile event was generated using the ALOS PALSAR data, acquired two weeks after the disaster. The ALOS AVNIR-2 data, acquired six months after the event, was also used. The image processing result using these data still showed damages in the affected area. This information indirectly conveys that post-disaster recovery was very slow in the Talcahuano area.

Community-based information is essential for disaster risk assessment, and participatory mapping activities such as DIG can cover the breadth of local spatial knowledge around a given area, including both quantitative and qualitative pieces of information [41]. Through this exercise it was possible to understand that general knowledge regarding the tsunami disaster had been well understood by the community. This result is reasonable considering the frequency with which communities in Chile have been affected by tsunami events.

Regarding the 2010 field survey report, it is estimated that at least a fourth of the total deaths were among the tourist population, who were in the coastal area for holidays. The local coastal communities, who experienced the 1960 earthquake and tsunami event, evacuated right after noticing the ground shaking [26]. This indicates that education and lesson from past events are highly valuable to mitigate tsunami fatalities. Nevertheless, tsunami countermeasures, including prevention systems, have not been developed adequately in Chile [26]. Additionally, there was a lack of emergency response staff in the field and lack of proper communication between the government and the population [8]. Therefore, it is necessary to constantly disseminate disasterrelated information through community-based activities, such as DIG.

Fundamentally, it is appropriate to extensively use qualitative data, such as local knowledge, to complement the use of quantitative data [23]. In order to improve risk assessments, it is important to combine geospatial knowledge with the local knowledge when assessing the risks of the tsunami inundation, which can then be combined to create a tsunami inundation zone map. Such a map can comprehensively communicate the risk of tsunami inundation. In the case of Talcahuano, tsunami inundation zones encompass high, medium, and low inundation level, which were delineated according to the DIG classification and the results of the satellite data analysis.

Concerning the result of the integrated tsunami risk assessment in Talcahuano area (Figure 15), the areas within the tsunami inundation zone, particularly the built-up area, are supposed to be developed by carefully taking into accuont DRR. The field observation report of the 2010 Chile event revealed that numerous houses at coastal locations (made of adobe, wood, and concrete) were built well enough to survive the earthquake, although the houses were destroyed by the tsunami that followed [28].

According to the visual interpretation of the VHR optical data (Figure 8) a housing area that was being constructed in the Salinas District on the pre-disaster image was damaged on the postdisaster image. A bridge in the area was also damaged, as shown in a field survey report [7]. In agreement with the tsunami inundation zone map (Figure 15), this area was located within the high inundation zone, highlighting the need for proper zoning laws.

This result points out the necessity to continuously improve the provision and dissemination of risk assessment information in the future. In this regard, satellite-based tsunami-damaged areas can be utilized as the reference for the future DIG activities. Further, the result of the DIG can be a useful input for improving satellite data analysis. Such a framework should allow for a continuous improvement of tsunami risk mitigation strategies, contributing to the sustainable development of disaster-prone areas.

\section{Conclusion}

Tsunami poses substantial risks to the sustainable development of coastal communities. In the present work the authors assessed the area of Talcahuano, Chile, which experienced massive casualties, economic loses and damage to infrastructure as a result of the 2010 tsunami event. The geospatial information on the extent of the damaged areas was effectively extracted by visually interpreting the VHR satellite optical data. The damaged areas were further assessed based on the NDVI difference between the pre- and postdisaster images of the ALOS AVNIR-2 satellite optical data. The total inundation area was extracted by subtracting the pre- from the post-disaster backscatter images of ALOS PALSAR. The total area of damaged correlated well with field observations, with an error of just $0.93 \%$. The damaged areas were also investigated according to the land classification, which showed a reduction in the extent of bare land, built-up, and vegetation areas due to the extent of the tsunami damage to such zones.

Additionally, information related to the tsunami inundation was collected by conducting a DIG, which encouraged community participation to map the disaster risks based on their own knowledge. The community evaluated well the exercise, and inundation lines and levels were obtained through the map created from the DIG.

Concerning the risk reduction for achieving sustainable development in such a disaster-prone area, an integrated risk assessment was carried out by compiling the disaster-related information based on the geospatial data and local knowledge. The result was represented as a map of the potential tsunami inundation zones (showing high, medium, and low inundation levels), which could be beneficial to disaster risk management efforts in the area, and improve the resilience of local communities to future tsunami events (for example by improving evacuation routes and sites).

\section{Acknowledgements}

This study was carried out by utilizing the ALOS data provided by JAXA through a joint research program with Yamaguchi University. The field survey report was facilitated by the Ingenieria Civil Oceania of the Universidad de Valparaiso in Chile. Moreover, a great appreciation is addressed to the reviewers for the paper refinement.

\section{References}

[1] Debarati G, Philippe H, and Regina B. Annual Disaster Statistical Review 2012: The Numbers and Trends. Belgium: Centre for Research on the Epidemiology of Disasters (CRED); 2012.

[2] ADRC. Total Disaster Risk Management: Good Practices. Japan: Asian Disaster Reduction Center (ADRC); 2005, p. 1-22.

[3] IOC. Hazard Awareness and Risk Mitigation in Integrated Coastal Area Management (ICAM). France: Intergovernmental Oceanic Commission (IOC) UNESCO Headquarters; 2009.

[4] Esteban M, Tsimopoulou V, Mikami T, Yun N. Y, Suppasri A, and Shibayama T. Recent Tsunamis Events and Preparedness: Development of Tsunami Awareness in Indonesia, Chile, and Japan. International Journal of Disaster Risk Reduction; 2013, 5: 84-97.

[5] UNISDR. UNISDR Global Assessment Report 2015 - GAR15: Tsunami Methodology and Result Overview. Revision 1. Norway: Geoscience Australia; 2015.

[6] Alarcon J. E. and Guillermo F. The 8.8 Magnitude Maule, Chile, Earthquake Seismological Review and Field Survey Observations. Trébol. Mexico: MAPFRE RE; 2010, XV (55): 5-17. 
[7] Koshimura S, Matsuoka M, Matsuyama M, Yoshii T, Mas E, Jimenez C, and Yamazaki F. Chile Earthquake and Tsunami Technical Report: Field survey of the 2010 tsunami in Chile. Japan: JST, JICA, and SATREPS Peru Project; 2010: 10-26.

[8] Siembieda W. and Guillermo F. Chile's 2010 M8.8 Earthquake and Tsunami: Initial Observations on Resilience. Journal of Disaster Research; 2010, 5 (5): 577-590.

[9] EM-DAT. 2015 Disasters in Numbers: The OFDA/CRED International Disaster Database. Belgium: Université Catholique de Louvain Brussels; 2016.

[10] UNISDR. Sendai Framework for Disaster Risk Reduction 2015-2030. Switzerland: United Nations International Strategy for Disaster Reduction (UNISDR); 2015.

[11] UNISDR. From a Reactive to Proactive then People Centered Approach to DDR: Taking Inspiration from the Hyogo Framework for Action to Implement the Sendai Framework for Disaster Risk Reduction. Geneva: United Nations International Strategy for Disaster Reduction (UNISDR); 2016.

[12] OOSA. The Value of Geoinformation for Disaster and Risk Management (VALID) Benefit Analysis and Stakeholder Assessment Vienna: United Nations Office for Outer Space Affairs (OOSA); 2013.

[13] Arthurton R, Raising Awareness of Marine-Related, Physical Hazards, and Risks in Coastal Management - an IOC-ICAM Project. United Kingdom: Coastal Geoscience; 2007.

[14] Joyce K.E, Wright K. C, Samsonov S. V, and Ambrosia V. G. Remote Sensing and The Disaster Management cycle. In: G. Jedlovec (Ed), Advances in Geoscience and Remote Sensing, Rijeka, Croatia: InTech; 2009, p. 317-346.

[15] Ramírez-Herrera M. T. and Navarrete-Pacheco J. A. Satellite Data for a Rapid Assessment of Tsunami Inundation Areas after the 2011 Tohoku Tsunami. Pure and Applied Geophysics; 2012, 168 (7): 11731328.

[16] Yamazaki F, Kouchi K, and Matsuoka M. Tsunami Damage Detection Using Moderate-Resolution Satellite Imagery. Proceedings of the $8^{\text {th }}$ U.S. National Conference on Earthquake Engineering; 2006.

[17] Chen S. and Motoyuki S. Tsunami Damage Investigation of Built-Up Areas Using Multitemporal Spaceborne Full Polarimetric SAR Images. IEEE Transactions on Geoscience and Remote Sensing; 2013, 51 (4): 1985-1997.

[18] Xhang Y, Jiang Z, Fang J. Y, and Cheng X. Analysis and Interpretation Of Tsunami Damage Caused by the 2011 Japan Earthquake Using ENVISAT ASAR images. Proceeding of the $35^{\text {th }}$ International Symposium on Remote Sensing of Environment, IOP Publishing; 2014.

[19] Gokon H, Koshimura S, and Matsuoka M. Object-Based Method for Estimating Tsunami-Induced Damage Using TerraSAR-X Data. Journal of Disaster Research; 2016, 11(2): 225-235.

[20] Matsuoka M. and Koshimura S. Tsunami Damage Area Estimation for the 2010 Maule, Chile Earthquake Using ASTER DEM and PALSAR Images on the GEO Grid. Proceeding of the $8^{\text {th }}$ International Workshop on Remote Sensing and Disaster Response; 2010.

[21] Chini M, Piscini A, Cinti F. R., Amici S, Nappi R, and De Martini P. M. The 2011 Tohoku (Japan) Tsunami Inundation and Liquefaction Investigated Through Optical, Thermal, and SAR Data. IEEE Geoscience and Remote Sensing Letter; 2013, 10 (2): 347-351.

[22] Hettiarachchi S. S. L, Samarawickrama S.P, and Wijeratne N. Risk Assessment and Management for Tsunami Hazard Case Study of the Port City of Galle. Bangkok: UNDP Asia-Pacific Regional Centre; 2011.

[23] Warner C. Participatory Mapping: a Literature Review of CommunityBased Research and Participatory Planning. Social Hub for Community and Housing, Faculty of Architecture and Town Planning, Technion; 2015.

[24] Komura T. Disaster Imagination Game (DIG) Manual for Nepalese People. Ministry of Home Affairs (MOHA) and JICA Study Team on KV EQ Disaster Mitigation Planning; 2002.

[25] Samaddar S, Chatterjee R, Misra B. A, and Tatano H. Participatory Risk Mapping for Identifying Spatial Risks in Flood Prone Slum Areas, Mumbai. Annuals of Disaster Prevention Research Institute, Kyoto University; 2011.

[26] Khew Y. T. J, Jarzebski M. P, Dyah F, San Carlos R., Gu J, Esteban M, Aránguiz R, and Akiyama T. Assessment of Social Perception on the Contribution of Hard-Infrastructure for Tsunami Mitigation to Coastal Community Resilience After the 2010 Tsunami: Greater
Concepcion Area, Chile. International Journal of Disaster Risk Reduction; 2015, 13: 324-333.

[27] Herd D.G, Youd T.Y, Meyer H, Arango C.J.L, Person W.J. The Great Tumaco, Colombia, Earthquake of 12 December 1979. Science; 1981, 221 (4481): 441-445.

[28] Shoji G, Pulido N, Sekiguchi T, Alva J, Lazares F. and Saito T Damage Investigation of the 2010 Chile Earthquake and Tsunami Consideration to the Damage of a Structure subjected to a Seismic Excitation and a Following Tsunami Wave Load. Japan: JST, JICA, and SATREPS Peru Project; 2010, p. 27-31.

[29] UNISDR. Disaster Reduction and Sustainable Development: Understanding the Links between Vulnerability and Risk Related to Development and Environment. Switzerland: United Nations International Strategy for Disaster Reduction (UNISDR); 2003.

[30] Maurer, T. (2013). How to Pan-Sharpen Images Using the gramSchmidt Pan-Sharpen Method- A Recipe. International Archives of the Photogrammetry, Remote Sensing and Spatial Information Sciences; 2013, XL-1(W1): 239-244.

[31] Liew S. C. Principles of Remote Sensing. Singapore: Center for Remote Imaging, Sensing, and Processing (CRISP), National University of Singapore; 2001.

[32] Bouvet M, Chander, Gorvl P, Santer R, and Saunier. Preliminary Radiometric Calibration Assessment of ALOS AVNIR-2. Geoscience and Remote Sensing Symposium, Barcelona: IGARSS; 2007, p. 26732676.

[33] Sah A. K, Sah B. P, Honji K, Kubo N, and Senthil S. Semi-Automated Cloud/Shadow Removal and Land Cover Change Detection Using Satellite Imagery. International Archives of the Photogrammetry, Remote Sensing and Spatial Information Sciences, Australia: XXII ISPRS Congress; 2012, XXXIX (B7): 335-340.

[34] Hansen, M. C, De Fries R. S, Townshend J. R. G, and Sohlberg R, Global Land Cover Classification at $1 \mathrm{~km}$ Spatial Resolution Using a Classification Tree Approach. International Journal of Remote Sensing; 2000, 21: 1331-1364.

[35] McCandless S. W. Jr. and Jackson C. R. Principles of Synthetic Aperture Radar. United States of America: Radar Imaging Resources; 2004, p. 1-23.

[36] Lillesand T. M. and Kiefer R. 1993. Remote Sensing and Image Interpretation. $3^{\text {rd }}$ Edition. New York: John Wiley and Sons; 1993.

[37] Curlander, J. C. and McDonough R. N. Synthetic Aperture Radar: System and Signal Processing. New York: John Wiley and Sons; 1991.

[38] Matsuoka, M. and Yamazaki F. Use of Interferometric Satellite SAR for Earthquake Damage Detection. Proceedings of the $6^{\text {th }}$ International Conference on Seismic Zonation. California; 200o, p. 1215 .

[39] Comuna de Talcahuano. Carta de Inundación por Tsunami Referida a Evento del 27 de Febrero 2010. Centro de Información Territorial de la Defensa Nacional Para la Región del Bio Bio; 2010 (in Spanish).

[40] Sistema de Informacion Comunal. Histórico Tsunami. http://riesgo.talcahuano.cl. Accessed 1 June 2017 (in Spanish).

[41] European Commission. Commission Staff Working Paper: Risk Assessment and Mapping Guidelines for Disaster Management Brussels: The European Comission; 2010.

[42] Bretschneider C., L, and Wybro P.G, Tsunami inundation prediction. Proceedings of the 15th ASCE Conference on Coastal Engineering; 1976, p. 1006-1024 